\title{
Las TIC como didáctica para el mejoramiento de la inferencia lectora
}

Sandra Milena Leandro Ayala * María Consuelo Yaya Blanco*:

Artículo de investigación

Fecha de Recepción: 30 mayo 2018.

Fecha de Aprobación: 14 septiembre 2018.

\section{Resumen}

Los resultados arrojados por el índice sintético de calidad de la Institución Educativa (I. E.) San Nicolás, en el año 2014, dio paso a la elaboración de una investigación para determinar las causas de los bajos desempeños de los estudiantes en algunas áreas del conocimiento; durante este proceso, se evidencia que la mayor dificultad que presentan los estudiantes en dichas pruebas se centra en la comprensión lectora. Para abordar esta dificultad, se planteó un objetivo general basado en la construcción de estrategias didácticas con herramientas TIC que aporten avances en los procesos de inferencia lectora en los estudiantes de educación básica. La investigación es de tipo Acción Educativa bajo el paradigma sociocrítico, tomando como referente a Restrepo (2004) quien plantea 3 fases en la investigación: deconstrucción, reconstrucción y validación. La metodología desarrollada bajo el enfoque Cualitativo en donde se toma la realidad del contexto tal y como sucede en su ámbito natural, obteniendo y analizando los fenómenos de acuerdo con las personas implicadas (Blasco y Pérez, 2007). La investigación se encuentra en la etapa final donde se realizó análisis de la observación documental y utilización de instrumentos, tales como: pruebas diagnósticas, diarios de campo y grupos focales.

Palabras clave: comprensión, estrategias, lectura, motivación, Tic.
* Institución Educativa San Nicolás, Boyacá (Colombia) samiazul1403@hotmail.com ** Institución Educativa San Nicolás, Boyacá (Colombia) m.yayabla@gmail.com 


\section{Introducción}

La lectura es una de las principales fuentes de adquisición del conocimiento, esta permite explorar mundos desconocidos e indagar y profundizar más en los mundos ya vistos o ya estudiados; los niños y niñas, en su etapa de crecimiento, son ávidos de nuevas e interesantes experiencias que, día a día, puedan llegar a ellos. Sin embargo, las nuevas tecnologías han incluido numerosas formas de explorar el mundo de manera más fácil, mucho más interactivas y palpables; es común ver videos en portales informáticos que resumen y muestran la información completa de una temática en especial, dejando a un lado los textos destinados para tal fin.

Es significativo generar hábitos lectores en los estudiantes desde las primeras etapas de vida de los niños, pues estos permitirán facilitar la adquisición del conocimiento durante todo el proceso académico. Por esta razón, se interviene el grado $2^{\circ}$ de la I.E. San Nicolás (sede Siderúrgica) mediante una combinación de lectura y TIC, en la cual se pretende mejorar la inferencia lectora, la cual representa un subnivel de una de las categorías análisis: "el lector".

La propuesta surge de las necesidades encontradas a partir de un análisis sistemático mediante la revisión de documentos Institucionales, resultados del Índice sintético de calidad y mediante el diseño y utilización de diferentes instrumentos para identificar las fortalezas y debilidades, donde se ve reflejada la dificultad que persiste en los estudiantes de básica de la I.E. San Nicolás en la competencia comunicativa, componente semántico nivel inferencial.

Se toma como punto de partida el ¿Cómo algunas estrategias didácticas basadas en aplicaciones informáticas aportan al proceso de inferencia lectora en los estudiantes de grado $2^{\circ}$ de la Institución Educativa San Nicolás sede Siderúrgica de Tuta?

La lectura es un tipo de comunicación, donde según Aventín (2004), "un emisor tiene un mensaje en mente, algo que quiere compartir; ya puede ser un sentimiento, una idea, un hecho, etc. Para poder transmitirlo ha de ponerlo en palabras, esto es, codificarlo y el lector debe descodificarlo mediante procesos mentales" (p. 6). Es entonces donde ese lector se encuentra contemplado.

Al verificar los teóricos que han abordado estos temas, se ve que la comprensión lectora ha sido considerada como la "esencia de la lectura" (Durkin citado en Madero \& Gómez, 2013, p. 115). Por otra parte, las etapas de la comprensión lectora (antes, durante y después de la lectura), según Solé, Miras \& Castells (2006), son estrategias importantes e imprescindibles en la enseñanza de la lectura en los estudiantes, para que estos tengan la capacidad de hacer interpretaciones más coherentes.

Metodológicamente, se aborda el proyecto desde los planteamientos de Restrepo (2004) en el que hace referencia al tipo de Investigación Acción Educativa 
(IAE), quien propone tres momentos: deconstrucción, reconstrucción y validación. Es así como se inicia con la deconstrucción a partir de grupos focales y distintos instrumentos, que permitieron identificar elementos para diseñar el material para la intervención en la etapa de deconstrucción y permitir evaluar los resultados.

Basadas en lo anterior, se opta por aplicar una estrategia didáctica apoyada en la herramienta informática de Author Edilim, que permite la presentación de lecturas cortas acompañadas de actividades tendientes a incentivar la comprensión lectora y la inferencia mediante la utilización de cuestionarios y juegos, acercando al estudiante a los avances informáticos de esta época $y$, a su vez, potenciando sus niveles de motivación en lo que a la lectura se refiere.

Para mejorar los procesos lectores, es necesario la aplicación de nuevas estrategias didácticas en los aprendizajes, teniendo en cuenta que los niños tienen mayor capacidad de análisis y comprensión cuando visualizan o hacen trabajos participativos (Orrego \& Toro, 2014), donde tienen la posibilidad de exponer sus propios puntos de vista, analizar las situaciones y comprobar a través de socializaciones; todo esto, basados en las afirmaciones de Dale (1969) en el gráfico conocido como Cono del Aprendizaje, el cual muestra el porcentaje de interpretación que tienen los estudiantes cuando visualizan e interactúan, señala qué aprendemos, y recordamos más acerca de lo que hacemos y decimos.
La utilización de didácticas con herramientas informáticas, permite determinar si estas pueden elevar en el estudiante los niveles de inferencia a la hora de realizar lecturas, aportando de esta forma hábitos lectores en los estudiantes de este nivel escolar. Se pretende, igualmente, familiarizar al estudiante y usar las tecnologías con las que cuenta la institución, algo favorable por el contexto rural en el que se encuentran los niños.

\section{Lectura, inferencia y dificultades}

El Gobierno nacional se ha puesto como meta lograr que Colombia sea catalogada como la mejor educada en Latinoamérica para el año 2025, para esto se han creado políticas educativas tendientes a mejorar los niveles académicos de los estudiantes y el desempeño de las pruebas externas de los mismos. En estas políticas educativas creadas para tal fin, se encuentra El Índice Sintético de Calidad (ISC), liderado por el Ministerio de Educación Nacional (MEN), el cual posiciona las Instituciones Educativas mediante la asignación de calificaciones obtenidas por ítems como el desempeño académico.

Para el año 2014, los resultados arrojados para la I.E. San Nicolás en lo que respecta al Índice Sintético de Calidad, fueron relativamente bajos, como consecuencia del desempeño en las pruebas externas de los estudiantes. Dentro de las observaciones entregadas a la I.E. en los resultados de las pruebas externas (Pruebas Saber) se resaltan las
El Gobierno nacional se ha puesto como meta lograr que Colombia sea catalogada como la mejor educada en Latinoamérica para el año 2025 
debilidades que posee la institución en lo concerniente a comprensión lectora, específicamente en lo que corresponde a la inferencia.

Como parte del mejoramiento institucional, se decide intervenir el grado $2^{\circ}$ de primaria, para determinar el nivel de comprensión lectora de los estudiantes, con el fin de proyectar para el año 2018 un buen desempeño en las Pruebas Saber grado $3^{\circ}$ y mejorar, a su vez, el índice sintético de calidad de la institución. Se toma este grupo a manera de grupo focal ya que, según Kragan (1991), "el grupo focal es un método cualitativo de investigación, que utiliza la discusión grupal como técnica para la recopilación de la información”. (p12) Se inicia un análisis de los mismos mediante la aplicación de una prueba diagnóstica tendiente a determinar el nivel de inferencia que estos poseen en su desempeño lector, entendiéndose así la inferencia como la manera que se busca preguntar, de diversas formas, algo; y que el estudiante logre obtener la respuesta a dichos interrogantes. Esta prueba, diseñada para determinar el nivel de comprensión lectora, tuvo como eje central "la inferencia" la cual es fundamental para el desarrollo del proyecto como tal.

La prueba diagnóstica se realiza mediante el desarrollo de una lectura, seguida de un cuestionario de seis preguntas relacionadas con la misma. Esta prueba se aplica a los estudiantes como parte del mismo proceso formativo, con el fin de analizar el comportamiento de los niños y niñas en su diario vivir "bajo el enfoque
Cualitativo en donde se toma la realidad del contexto tal y como sucede en su ámbito natural, obteniendo y analizando los fenómenos de acuerdo con las personas implicadas" (Blasco y Pérez, 2007: p25). De este cuestionario, la pregunta número 6 fue la única proyectada a determinar la capacidad inferencial de los estudiantes en lo que a lectura respecta, arrojando como resultado:

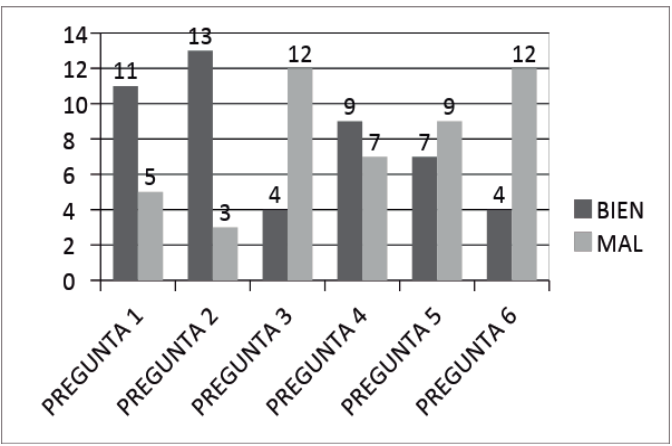

Figura 1. Prueba diagnóstica, estudiantes grado $2^{\circ}$.

Fuente: las autoras.

La prueba diagnóstica arroja un porcentaje muy bajo de estudiantes con capacidad de inferencia dentro del desarrollo lector, pues solamente 4 de 16 estudiantes respondieron acertadamente dicha pregunta, es decir, el $75 \%$ de los estudiantes no poseen capacidad inferencial.

Existen factores que impiden que los estudiantes posean buenos niveles de desempeño en su proceso lector y entre estos factores se resaltan los de tipo motivacional, pues al estudiante poco le gusta la lectura. Para dar solución a esta problemática lectora 
de los estudiantes, se interviene el curso mediante la utilización de la metodología IAE, partiendo de las etapas planteadas por Restrepo (2004).

Para ITESM (2010), "La estrategia didáctica es el conjunto de procedimientos, apoyados en técnicas de enseñanza, que tienen por objeto llevar a buen término la acción didáctica, es decir, alcanzar los objetivos de aprendizaje" (p. 192). En este caso, la estrategia didáctica está referida a la aplicación de sesiones de lectura acompañadas de actividades de tipo lúdico mediante la mediación de las TIC, dicha mediación comprende la utilización del programa "Author Edilim”, el cual brinda la posibilidad de presentarle a los estudiantes una lectura con una presentación atractiva, permite insertar juegos de rompecabezas, concéntrese, sopas de letras, juego de parejas y cuestionarios con preguntas de tipo abierta o de opción múltiple con única respuesta, con el fin de mejorar la inferencia lectora de los estudiantes.

La intervención es de tipo TIC introduciendo el concepto de Tecnología Educativa, definida como el medio que establece un camino entre las ciencias educativas (psicología, pedagogía, filosofía, y otras) y sus aplicaciones para resolver problemas de aprendizaje (Riveros, Gómez \& Abrego, 2013), donde el problema se centra en la lectura, en especial, la inferencia; y el camino es tomado como una conexión entre el texto-contexto-lector y "Author Edilim" no es más que un mediador que permite que estos tres elementos se relacionen con facilidad dentro del proceso lector como tal (Muñoz, 2016).

\section{Deconstrucción y reflexión metodológica}

Para Solé (1992), “conseguir que los alumnos aprendan a leer correctamente es uno de los múltiples retos que la escuela debe afrontar" (p. 27), pero estos retos no son otra cosa que la oportunidad de innovar en nuevas metodologías ligadas a los avances tecnológicos de nuestra época. Para Cuervo (2017): "uno de cada tres menores de 12 años en Colombia es usuario permanente de Internet, y el $40 \%$ de los bebes (sic) entre dos y cuatro años tienen contacto esporádico con dispositivos móviles" (párr. 4).

Según la experiencia profesional de las autoras, el docente ve el proceso lector como una relación entre los estudiantes y los diferentes tipos de texto, resaltando la importancia de los libros y buscando que los niños y niñas se acerquen cada vez más a los mismos, dejando de lado los gustos y motivaciones de nuestros educandos. Muchas veces, se olvidan detalles simples, como el hecho de que los niños constantemente usan el dispositivo móvil de sus padres, el cual manipulan, juegan y realizan acciones que estos permiten hacer.

Como proceso deconstructivo tendiente a mejorar la capacidad lectora de nuestros estudiantes en lo que a inferencia se refiere, se ha tomado la decisión de dejar por un tiempo de lado la lectura mediante la utilización de libros, cambiando esta metodología por actividades más amigables con los
Para Solé (1992), “conseguir que los alumnos aprendan a leer correctamente es uno de los múltiples retos que la escuela debe afrontar" 
niños y niñas, haciendo referencia a la primera etapa de la IAE en la que el docente analice su quehacer pedagógico y determine los elementos que le son o no favorables, buscando las fortalezas y debilidades donde se critique a fondo y genere una transformación en su práctica (Restrepo, 2004).

\section{Reconstrucción y tic}

La reflexión respecto a la práctica pedagógica en lo que respecta a la lectura, da espacio para el segundo paso dentro de la I.A.E. de Restrepo (2004): “La reconstrucción” en donde se hace necesario el planteamiento de un nuevo proceso o estrategia que permita el mejoramiento a corto o largo plazo en lo que se refiere a la inferencia lectora. Tomando como referencia uno de los procesos en la comprensión lectora, según Gagné (citado en Jiménez Rodríguez, 2004), la comprensión inferencial: es cuando el lector realiza deducciones de lo que está escrito de forma explícita, aquello que está implícito, bien porque el autor no puede o no quiere decirlo; procesos de integración: relaciona oraciones que entre ellas no guardan relación alguna; procesos de resumen: llevadas a cabo con las ideas principales del texto y, por último, los procesos de elaboración: ayudado por los conocimientos que se tienen en donde se elabora una representación relacionado con el significado.

Cabe recordar que, para Ripoll (2015),

Se ha comprobado cómo las clasificaciones que se han realizado hasta el momento han utilizado nombres como inferencias lógicas o inferencias pragmáticas para denominar a conceptos distintos. Esto también sucede con otros nombres, como inferencias elaborativas o conectivas. Para evitar perpetuar esa confusión la clasificación que se va a proponer utiliza una denominación neutra: inferencias de tipo I, II, III, IV y V. (p. 111).

Inferencias de tipo I: el lector responde a preguntas como "¿a qué (o a quién) se refiere?", “¿de qué (de quién) habla el texto cuando dice...?”.

Inferencias de tipo II: el lector responden a preguntas como "¿por qué?” o “¿qué relación hay entre... y...?”. En este tema, su empleo es dar conexión al texto estableciendo relaciones causaefecto que no están explícitas en él. Según Ripoll, las inferencias de este tipo realizadas en los textos narrativos, son relacionadas con las emocionalidades de los personajes (envidia, venganza, altruismo, indignación).

Inferencias de tipo III: para este tipo de inferencia, las preguntas son "¿qué sucederá?”, "¿qué se puede predecir sabiendo que...?” o “¿para qué?”. La situación principal de las inferencias de tipo III es hacer hipótesis sobre los sucesos del texto.

Inferencias de tipo IV: este tipo de inferencias es el más vago de todos y responde a preguntas como "¿cuándo?”, “¿dónde?”, “¿cómo?”, “¿con quée?”, “¿de qué color?” y muchas otras de ese tipo. Se podrían englobar en una pregunta general, que sería: “¿qué más se puede decir sobre esto?”. 
Inferencias de tipo $\mathrm{V}$ : responden a preguntas como "¿qué me están contando aquí?" o "¿qué quiere decir todo esto?”. Donde la relación con el texto es explícita y el lector debe dar conocimiento de lo que no se ve en el mismo.

Hay tres elementos fundamentales que deben ser tenidos en cuenta en el momento de realizar la reconstrucción como tal: El texto, el contexto y el lector. Para Hurtado (2005),

Lo que determina al texto no es la extensión sino la intención comunicativa, la cual está, a su vez, determinada por la manera como las oraciones se relacionan entre sí hasta construir el hilo argumental del tema. Cuando se concibe el texto de esta manera, se identifican también diferentes factores que facilitan u obstaculizan su comprensión por parte del lector, que van desde el contenido, o el vocabulario, hasta la forma como está redactado. (p. 7).

El contexto estipulado como el entorno que permite o no el buen desarrollo de la lectura y, finalmente, el lector es quien mediante el proyecto se encuentra en la etapa de intervención, es este lector quien puede inferir y el que debe y puede mejorar con la aplicación de las TIC. Consideramos entonces estos tres aspectos de la lectura como las categorías de análisis, y es de allí de donde se extrae uno de los subniveles vistos como punto central de nuestra investigación: la inferencia, la cual es derivación de la categoría "lector".

La estrategia didáctica que se tiene en cuenta es la utilización de las herramientas tecnológicas con las que se cuenta en el aula. Según Dale (1969), en el gráfico conocido como cono del Aprendizaje, muestra el porcentaje de interpretación que tienen los estudiantes cuando visualizan e interactúan; señala que aprendemos y recordamos más, acerca de lo que hacemos y decimos. Por otra parte, la Fundación Telefónica en el desarrollo de los programas sobre cualificación docente en TIC (SCOLARTIC), socializa que independientemente de la materia que se quiera enseñar, el docente debe reflexionar sobre: ¿qué motiva a los estudiantes?, ¿cómo movilizarse al cambio y transformación del aula?, ¿cuáles son los métodos que se implementa para esta transformación? ya que todos los seres humanos tienen las habilidades de creatividad e innovación, la capacidad de aprenderlas, desarrollarlas y fortalecerlas a medida que se utilizan.

El proceso de reconstrucción parte de la utilización de las herramientas tecnológicas con las que cuenta la institución: las Tablet donadas por el ministerio de las TIC y los equipos portátiles, los cuales son suministrados por el programa computadores para educar. Estos equipos tecnológicos brindan posibilidades diferentes que permiten aportar, en gran parte, herramientas que potencian el buen desempeño de los estudiantes en su quehacer pedagógico, resaltando que "Los ambientes computacionales tienen el potencial para aumentar el aprendizaje de los estudiantes" (Leguizamón, 2010, p. 5).
Lo que determina al texto no es la extensión sino la intención comunicativa, la cual está, a su vez, determinada por la manera como las oraciones se relacionan entre sí hasta construir el hilo argumental del tema. Cuando se concibe el texto de esta manera, se identifican también diferentes factores que facilitan u obstaculizan su comprensión por parte del lector, que van desde el contenido, o el vocabulario, hasta la forma como está redactado. 
Como estrategia didáctica, se optó por la utilización de una aplicación en los equipos de cómputo, la cual permite a los estudiantes interactuar y resolver actividades mediante el juego y la manipulación del equipo informático. Esta aplicación llamada "Author Edilim”, permite al docente elaborar actividades que tienen, como punto inicial, la lectura; seguida de esta, el programa permite la interacción de los estudiantes mediante el desarrollo de pruebas didácticas como rompecabezas, sopas de letras, cuestionarios, parejas de palabras y demás.

Aunque muchas veces se ve el juego como una barrera para el aprendizaje, es importante tener en cuenta la apreciación de Leyva (2011), donde nos plantea que

Con lo que refiere entonces al considerar al juego como una estrategia didáctica es importante comprender, que "se establece una red de relaciones entre los participantes de la actividad (niños $\mathrm{y}$ docentes considerados en forma individual como grupo-clase), los instrumentos o artefactos mediadores (objetos o juguetes), los roles y las reglas de acción, y el objeto de conocimiento (contenidos de enseñanza). Esta nueva red, particulariza y dinamiza las prácticas de enseñanza" (pp. 1415) La tecnología, y en especial la informática, poseen instrumentos y aplicaciones con especificaciones atractivas al gusto de la mayoría de los estudiantes. La elaboración de las actividades lectoras mediante el uso de las TIC, ha permitido que los alumnos escalen positivamente en un proceso de mejoramiento de su proceso lector, el cual inicia para nuestro caso con un elevado promedio de desaciertos, los cuales fueron contados como intentos para disminuir el impacto que genera el error en el estudiante.

La primera actividad denominada sesión uno y diseñada mediante la utilización de la aplicación "Author Edilim”, es el punto de partida del encuentro de los estudiantes de la I.E. San Nicolás y las TIC para fortalecer el proceso lector. Las expectativas fueron grandes y se destaca el hecho de que los estudiantes buscaron como primera instancia la elaboración de las pruebas pasando por alto la lectura, aunque durante el desarrollo de dichas pruebas, fue necesario retomar la misma para desarrollar los puntos que requerían información del texto escogido.

Para Leguizamón (2010), “Aprender en los ambientes computacionales le exige a un aprendiz que regule su aprendizaje" (p. 5), y es allí donde se destaca la importancia de las TIC en el aula y es este el punto de partida en el proceso de reconstrucción de la enseñanza en lo que a la lectura se refiere. Generalmente, en el aula de clases es el docente quien impone los tiempos y las secuencias para el desarrollo de las unidades, pero en este caso es el estudiante quien debe proponer sus propios ritmos de acuerdo con las necesidades que encuentra durante el desarrollo de las actividades propuestas.

Hay que tener en cuenta que el proceso de reconstrucción planteado en el desarrollo del proyecto, tiene como fin 
principal el potenciar las habilidades lectoras de los estudiantes de la I.E. San Nicolás en lo que a inferencia se refiere, no es el fin hacer a un lado los textos, los cuales consideramos como fuente importante del conocimiento y compartimos la opinión de Gómez (2016), quien considera que:

no querer abolir la escritura con lápiz y papel, ni tampoco siquiera contemplar la idea de que la lectura en libros impresos deba desaparecer, mucho menos pensar que se es más inteligente si estas prácticas se hacen en un computador, una tableta o un celular (p. 61).

Lo que se pretende es mejorar y potenciar las capacidades de los estudiantes en lectura, mejorando su índice motivacional y, en sí, generando el hábito lector.

Luego del análisis y de los diferentes parámetros tenidos en cuenta durante el proceso de reflexión, se determina que la intervención para el proceso de lectura mediante el apoyo con TIC, debe hacerse con la aplicación de 4 lecturas, divididas en sesiones y acompañadas de actividades prácticas e interactivas. Estas actividades desarrolladas y con separación de tiempo igual a una semana, permiten al estudiante familiarizarse con el proceso lector y obtener resultados que se mostrarán más adelante.

\section{Resultados y validación}

Se hizo una intervención del grupo dividida en cuatro sesiones de trabajo de lectura, tendientes a mejorar el nivel de inferencia de los estudiantes en su proceso lector y, a su vez, incentivar el gusto por la lectura. Las sesiones fueron planteadas con actividades de cuatro o cinco puntos de tipo interactivo, dentro de las cuales un punto estaba destinado a afianzar el nivel de inferencia de los estudiantes.

Los diferentes puntos no inferenciales son de tipo lúdico, compuestos por rompecabezas, sopas de letras, juegos de parejas, juegos de concéntrese, con altos contenidos gráficos relacionados con el texto propuesto para la actividad; los textos escogidos son de tipo narrativo con lecturas infantiles acorde a la edad de los estudiantes de grado $2^{\circ}$. El punto inferencial es compuesto por una pregunta de tipo cerrada para cada una de las cuatro sesiones, donde el estudiante debe contestar acertadamente para que el programa le valide como completa su actividad.

Como elemento motivante, se adicionó a cada punto de las actividades una grabación de aplausos la cual solo suena en el momento que el estudiante termina, esto hizo que el estudiante se sintiera orgulloso de sí mismo y fuera felicitado frente a sus demás compañeros. Los resultados de las sesiones se muestran a continuación:

\section{Sesión 1:}

Lectura: Pipo busca un amigo, Número de Puntos: 4, Punto de tipo inferencial: P4, Medición de la actividad: Intentos, Resultados: (ver figura 2).
No querer abolir la escritura con lápiz y papel, ni tampoco siquiera contemplar la idea de que la lectura en libros impresos deba desaparecer, mucho menos pensar que se es más inteligente si estas prácticas se hacen en un computador, una tableta o un celular 


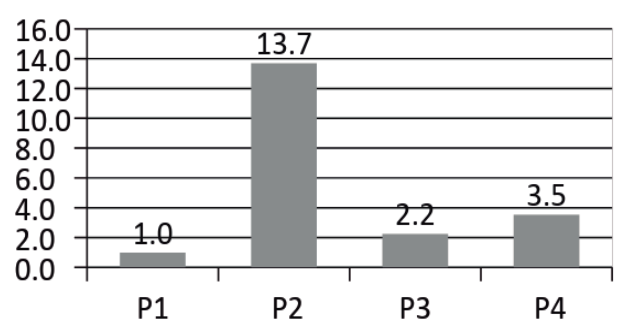

Figura 2. Promedio Intentos sesión 1 aplicación por punto "Author Edilim". Fuente: elaboración propia.

\section{Sesión 2:}

Lectura: La boda de Martha, Número de puntos: 5, Punto de tipo inferencial: P2, Medición de la actividad: Intentos. Resultados: (ver figura 3).

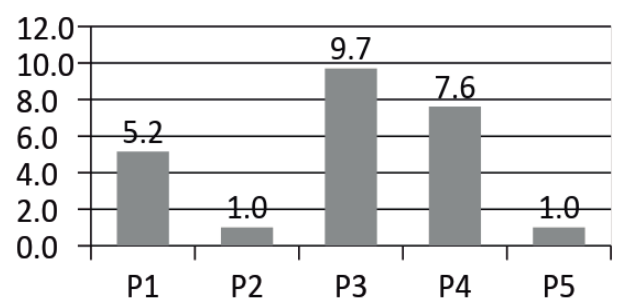

Figura 3. Promedio Intentos sesión 2 aplicaciones por punto "Author Edilim". Fuente: elaboración propia.

Sesión 3: Lectura: Caperucita roja, Número de puntos: 5, Punto de tipo inferencial: P3. Medición de la actividad: Intentos, Resultados (ver figura 4).

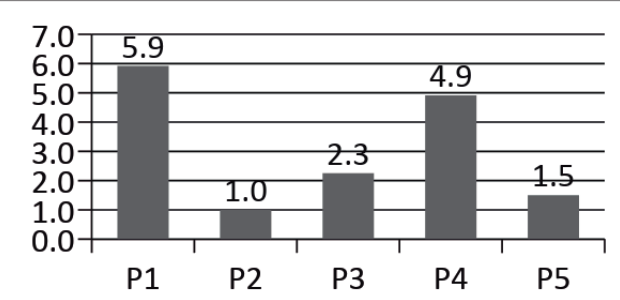

Figura 4. Promedio Intentos sesión 3 aplicaciones por punto "Author Edilim".

Fuente: elaboración propia.
Sesión 4: Lectura: Las hojas secas, Número de puntos: 4, Punto de tipo inferencial: P3, Medición de la actividad: Intentos, Resultados (ver figura 5).

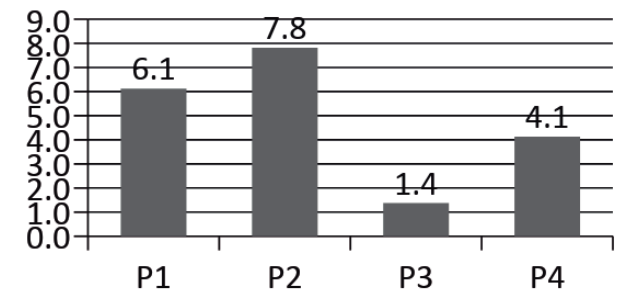

Figura 5. Promedio Intentos sesión 4 aplicaciones por punto "Author Edilim". Fuente: elaboración propia.

\section{Total intentos promedio}

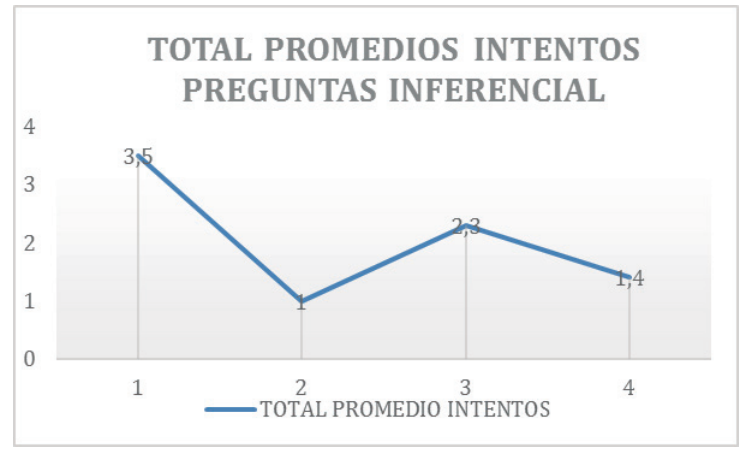

Figura 6. Consolidado intentos históricos puntos de inferencia en aplicación "Author Edilim".

Fuente: elaboración propia.

La prueba diagnóstico aplicada a los estudiantes de grado $2^{\underline{o}}$, arrojó porcentajes negativos en lo que respecta a los niveles de inferencia y en el proceso lector (ver figura 1). Pasada esta prueba, se intervino con cada una de las sesiones destinadas para tal 
fin, las cuales fueron documentadas en diarios de campo, donde registra aquellos hechos que son susceptibles de ser interpretados para la investigación realizada, teniendo en cuenta que para Porlán (1987) el diario es "una herramienta para la reflexión”p

Dentro de los primeros hallazgos, se destacan los altos niveles de motivación de los estudiantes a la hora de iniciar la actividad; dejar de lado los cuadernos para interactuar con los equipos informáticos, llena de expectativas al niño(a). La curiosidad es constante y las instrucciones que se realizan dentro de la clase tienen buena aceptación y rápida comprensión, permitiendo afirmar que la actividad, desde sus inicios, es un éxito por las ambiciones del estudiante por aprender.

En lo referente a texto-contextolector, la herramienta informática permite al estudiante relacionarse con mayor facilidad al contenido de la lectura, las actividades de cada sesión, más que medir lo comprendido del texto, aumentan en gran parte la concepción de lo que el autor pretende transmitir con la misma, los juegos de rompecabezas, de parejas, poseen altos contenidos gráficos relacionados con la lectura, lo que permite al niño(a) familiarizarse con mayor facilidad con el contexto en el que se desenvuelve la historia.

Generalmente, el contexto influye mucho en la efectividad de la lectura que se realiza; el ruido y los demás fenómenos externos, introducen interrupciones que muchas veces son inevitables. La aplicación de las actividades lectoras con mediación de las TIC, permitió que los niveles de atención de los niños en la lectura aumentaran, aunque los distractores propios de las clases no desaparecieron, la concentración en la actividad por parte de los estudiantes no disminuyó. El equipo informático se convierte en contexto y la concentración se ve como una nueva fortaleza de este tipo de lecturas, permitiendo al estudiante aislar de su mente los distractores del entorno.

En lo que respecta a la valoración de las actividades, no se tuvieron en cuenta errores y aciertos como tal, porque el estudiante debe culminar la totalidad de los puntos de la aplicación. Se muestra la cantidad de intentos que el estudiante hace para desarrollar cada uno de los puntos y estos varían dependiendo el tipo de actividad. Los intentos que realiza el estudiante en el punto inferencial, pueden tener múltiples interpretaciones que van desde el manejo del equipo informático hasta el poseer buenos niveles inferenciales.

En la primera sesión, se vio la apatía de los estudiantes por la lectura, pues estos iniciaron desarrollando los diferentes puntos pasando por alto la misma, sin embargo, al llegar al punto inferencial la necesidad de conocer el contenido del texto, los obligó a volver para saber la temática que deberían haber visto desde un inicio. De esto, los estudiantes aprendieron la importancia de la lectura; y para las siguientes sesiones, esta fue realizada desde el primer momento. 
Analizar el progreso del componente inferencial de la lectura en este proceso, puede confundirse con el nivel de manejo de la aplicación por parte de los estudiantes, sin embargo, el hecho de que el punto de inferencia corresponda a una pregunta de selección múltiple con única respuesta, brinda un amplio índice de confiabilidad en el estudio. De esto, podemos deducir que se muestra un avance de los estudiantes en la solución de preguntas de tipo inferencial, pues inicia de un promedio de 3,5 intentos para resolver la pregunta (figura 5) hasta llegar a un promedio de 1,4 en la sesión número 4. Esto demuestra que la evolución ha sido constante, pues cada vez fallan menos; los estudiantes han mejorado su lectura y la aplicación ha demostrado que puede ser un factor motivante para ellos.

Al mostrar un avance en el proceso inferencial de los estudiantes, podemos afirmar quela incursión de las TIC es una herramienta apta para la consecución de los objetivos planteados por las docentes en el inicio del proyecto, las actividades demostraron al estudiante la importancia que tiene la lectura en cualquier proceso que se quiera aplicar, mostraron que leer y conocer un texto proporciona herramientas que mejoran su proceso, que potencia su análisis y que, en definitiva, mejora su desempeño como estudiante.

Hasta el momento, es difícil determinar cuánto ha mejorado el proceso lector con actividades escritas y lecturas no elaboradas o presentadas con herramientas TIC, pues ese punto no ha sido abordado aún, los resultados serán vistos más adelante y presentados en la sustentación final de la tesis Incidencia de las Tic en el Proceso de Inferencia en la Comprensión Lectora, ya que aquí se muestra los resultados de las dos primeras fases del proyecto. Lo que sí se puede afirmar, es que los estudiantes han mejorado su desempeño en lo que respecta a la inferencia en la lectura con la utilización de herramientas tecnológicas, se ha observado el alto grado de interés de los niños y niñas por la lectura.

La aplicación “Author Edilim” logró convertirse en un enlace entre textolector, desde el momento que el estudiante inicia a afianzar el contenido de la lectura mediante la relación de las imágenes con el contenido del relato; lector-contexto, cuando el estudiante aísla las interrupciones generadas por el ambiente escolar y se centra en su actividad en el equipo informático, convirtiendo el computador en su nuevo contexto; finalmente, aunque en las actividades relacionan mucho el texto con el contexto, por ahora se considera que esta relación no determina factores importantes para el estudio, el cual se centra en la inferencia, relación directa entre el lector y el texto.

\section{Conclusiones}

La mediación TIC permite introducir en el proceso lector, un conector entre texto, contexto y lector, el cual proporciona herramientas que mejoran la inferencia en la lectura por parte de los estudiantes de grado segundo, 
este conector es la aplicación "Author Edilim" y las actividades elaboradas por las docentes, aunque es importante trabajar para que este conector se consiga sin necesidad de usar medios tecnológicos, debido a la importancia que tienen los libros en los diferentes ámbitos académicos.

Las competencias inferenciales de los estudiantes mejoraron con la inclusión de la tecnología en el proceso lector, lo que indica que las TIC aportan altos niveles motivacionales en los procesos educativos; además de esto, la constante manipulación de los elementos informáticos mejora las habilidades de los niños y niñas en el uso de los equipos de cómputo, haciendo que el progreso sea trasversal con las diferentes áreas del conocimiento.

Los dispositivos electrónicos, en especial los celulares, poseen aplicaciones que permiten interactuar, jugar, conocer, entre otras, lo que genera una constante absorción del tiempo del individuo y un alejamiento del mismo de los instrumentos que, por décadas, hemos usado para la formación dentro de las instituciones educativas (textos). Debido a esto, se puede concluir que el docente no debe ser un obstáculo para el crecimiento o el avance de la tecnología y de la sociedad como tal, el docente no debe alejar al estudiante de las posibilidades de estar a la vanguardia del nuevo conocimiento, la tarea del docente debe ser guiar al estudiante para que este dé un buen uso de las nuevas tecnologías $\mathrm{y}$, a su vez, sea capaz de crear en este tipo de dispositivos, herramientas que le sirvan para su crecimiento personal y su avance en el proceso formativo y académico.

Las actividades lúdicas y los juegos aportan altos niveles motivacionales en los diferentes procesos en los que se relaciona el ser humano, si utilizamos las ventajas que nos proporcionan dichas actividades y le sumamos las mejoras que nos aportan los elementos informáticos $\mathrm{y}$, a su vez, lo combinamos a los procesos académicos, podemos obtener buenos desempeños en nuestros estudiantes e igualmente nuevas experiencias en nuestra labor docente como tal, por eso es importante darnos la oportunidad de aplicar nuevas experiencias tendientes a mejorar el proceso educativo de nuestras instituciones escolares.

El replanteamiento de las metodologías de la enseñanza por parte de las docentes, permitió adquirir destrezas de tipo tecnológico, las cuales se ajustan al pentágono denominado: pentágono TIC del Ministerio de Educación Nacional, el cual nos muestra las competencias tecnológicas que debe poseer un docente, donde este debe ser explorador, integrador e innovador, competencias que se refuerzan y se estimulan con el desarrollo del proyecto y que indirectamente fortalecemos los requerimientos allí planteados, lo que muestra que no solo los estudiantes están recibiendo beneficio de las actividades aplicadas, pues también el docente puede mejorar su práctica y su desempeño profesional. 


\section{Referencias bibliográficas}

Aventín, S. (2004). Comunicación, expresión y lenguaje. Recuperado de: aprendeenlinea.udea.edu.co/1ms/men_udea/mod/resource/view. php?id=16973

Blasco, J., \& Pérez, J. (2007). Metodologías de Investigación en las Ciencias de la Actividad Física y el Deporte: Ampliando Horizontes. Recuperado de https:// rua.ua.es/dspace/bitstream/10045/12270/1/blasco.pdf

Cuervo, J. (2017, 4 de mayo). Niños y tecnología ¿Oportunidad o amenaza? El Tiempo. Recuperado de http://www.eltiempo.com/tecnosfera/dispositivos/ relacion-entre-los-ninos-y-la-tecnologia-84130

Dale, E. (1969). Audiovisual methods in teaching. Dryden Press. Londres

Gómez, S. (2016). Las Tic en la Enseñanza-Aprendizaje de Lectura y Escritura en Lengua Castellana. Santiago de Cali: Universidad de San Buenaventura.

Hurtado, R. (2005). Hacia la comprensión de la lectura. En Universidad de Antioquia, Lineamientos curriculares. Lengua Castellana (pp. 72-74). Medellín: Universidad de Antioquia.

ITESM. (2010). Dirección de investigación y desarrollo. Capacitación en estrategias y técnicas didácticas. Recuperado de http://www.itesm.mx/va/dide/documentos/infdoc/ Est_y_tec.PDF.

Leguizamón, M. (2010). Proyectos Tecnológicos de Aula: una Estrategia Colaborativa Apoyada en las TIC. En Encuentro Nacional de Investigación y Desarrollo. ENID 2010. Septiembre, Bogotá.

Leyva, A. (2011). El Juego como Estrategia Didáctica en la Educación Infantil. Bogotá: Pontificia Universidad Javeriana.

Madero, I., \& Gómez, L. (2013). El Proceso de Comprensión Lectora en Alumnos de Tercero de Secundaria. Revista Mexicana de Investigación Educativa, 18(56), 113-139. Recuperado de http://www.redalyc.org/pdf/140/14025581006.pdf

Muñoz, H. (2016). Mediciones tecnológicas: nuevos escenarios de la práctica pedagógica. Praxis \& Saber, 7(13), 199-221. https: // doi. org/10.19053/22160159.4172

Orrego, J., \& Toro, L. (2014). Relaciones vitales: el aula como escenario permanente de investigación. Praxis \& Saber, 5(10), 121-139. https: // doi. org/10.19053/22160159.3025 
Porlán (1987), aprendeenlinea.udea.edu.co/boa/contenidos.php/.../128/1/ contenido/

Restrepo, B. (2004). La investigación-acción educativa y la construcción de saber pedagógico. Educación y Educadores, (7), 45-55. Cundinamarca, Colombia: Universidad de La Sabana.

Ripoll Salcedo, J. C. (2015). Una clasificación de las inferencias pragmáticas orientada a la didáctica. Investigaciones Sobre Lectura, 4, 107-122. Recuperado de http://comprensionlectora.es/revistaisl/index.php/revistaISL/article/ view/43/pdf_14

Riveros, I., Gómez, M., \& Abrego, R. (2013). Tecnologías educativas y estrategias didácticas: criterios de selección. Revista Educación y Tecnología, (3), 190-206. Recuperado de https://www.researchgate.net/profile/Marcela_Gomez_ Zermeno/publication/266385186_Tecnologias_educativas_y_estrategias_ didacticas_criterios_de_seleccion/links/542ec1680cf277d58e8eeb21.pdf

Solé, I., Miras, M., \& Castells, N. (2000). La evaluación de la lectura y escritura mediante pruebas escritas en las etapas de educación primaria (6-12) y secundaria (12-16). Lectura y Vida, 21(3), 6-15.

Solé, I. (1992). Materiales para la innovación educativa. Recuperado de http:// courseware.url.edu.gt/DESAC1/Estudiantes/Lectura $\% 20 y \% 20$ Escritura $\% 20$ Acad\%C3\%A9micas/Estrategias\%20de\%20lectura\%20-020Isabel\%20Sole/ Isabel_Sole_Estrategias_de_lectura_pag_17_a_55\%5B1\%5D.pdf 